\title{
Carnets
}

Revue électronique d'études françaises de l'APEF

Deuxième série - 6| 2016

Exotopies de Barthes

\section{Barthes dans la théorisation du renouveau romanesque}

José Domingues de Almeida

\section{OpenEdition}

1 Journals

\section{Édition électronique}

URL : http://journals.openedition.org/carnets/827

DOI : $10.4000 /$ carnets.827

ISSN : 1646-7698

Éditeur

APEF

\section{Référence électronique}

José Domingues de Almeida, «Barthes dans la théorisation du renouveau romanesque », Carnets [En ligne], Deuxième série - 6 | 2016, mis en ligne le 31 janvier 2016, consulté le 19 avril 2019. URL : http:// journals.openedition.org/carnets/827 ; DOI : 10.4000/carnets.827

Ce document a été généré automatiquement le 19 avril 2019.

\section{(c) (i) (8)}

Carnets est mis à disposition selon les termes de la licence Creative Commons - Atribution - Pas d'utilisation commerciale 4.0 International. 


\title{
Barthes dans la théorisation du renouveau romanesque
}

\author{
José Domingues de Almeida
}

1 Le terme « intériorisation » sied à merveille pour préciser le rôle joué par Barthes dans le discours critique de la théorisation du renouveau romanesque français au tournant des années quatre-vingt. En effet, si le théoricien mutant et pluriel Roland Barthes a fait l'objet d'une intériorisation dans le métalangage narratologique auquel recourent les théoriciens de la littérature, il a également subi une appropriation à la faveur de la caractérisation de ce tournant. Il faut dire que son discours s'y prête, vu la reformulation qu'il a opérée de certains de ses concepts clés, ou encore la propension métaphorique, et dès lors moins objective -, de ses analyses.

2 Ceci dit, le renouveau romanesque devait occuper, autour de l'an 2000, une part considérable de la recherche en littérature. On ne compte pas les essais issus des thèses universitaires glosant les mécanismes intrinsèques de la réviviscence du texte fictionnel hexagonal (la fiction francophone, et allophone en français ne relevant pas du même diagnostic).

3 En amateur, nous tâcherons d'évoquer cette tranche infime de l'impact de Roland Barthes, cette fois sur les discours d'escorte de la fiction française dite encore, - mais pour combien de temps? - «contemporaine »; lui qui apparaît aujourd'hui comme un "signe» incontournable de notre culture littéraire, et de la culture tout court, dans l'acception qu'il a lui-même forgée de ce concept (Barthes, 1970).

Dans les essais, les thèses et les articles auxquels nous renverrons, nous nous rendrons à des évidences plutôt consensuelles quant aux mouvances et aux taxinomies de la fiction narrative dont a accouché ce tournant chronologique et critique: "minimalisme", "postmodernité", «écriture blanche», "nouvelle fiction", " nouveaux écrivains de Minuit », etc. En fait, les années soixante-dix avaient prolongé le travail de sape narratif entamé par les néo-romanciers en l'intensifiant dans un textualisme militant, mais en dégageant et affichant, justement, les spécificités de l'œuvre littéraire comme « texte », et de l'écriture en tant qu'espace paradoxal fait d'arbitraire et de liberté; ce que Le Degré 
zéro de l'écriture signalait en la plaçant à l'intersection des axes horizontal de la langue et vertical du style :

Langue et style sont des objets ; l'écriture est une fonction : elle est le rapport entre la création et la société, elle est le langage littéraire transformé par sa destination sociale, elle est la forme saisie dans son intention humaine et liée ainsi aux grandes crises de l'Histoire (Barthes, $1953: 24$ )

5 Cette phase textuelle de la théorisation littéraire, la recherche en littérature contemporaine la pose comme une transition nécessaire, parfois quelque peu extravagante, datée, logée entre le nouveau roman et le roman contemporain, dans lequel le référent revient, tout comme le sujet d'ailleurs. Dans cette optique, la hâte critique d'approcher, voire de caractériser un récit littéraire différent et novateur fait que l'on tende spontanément et péjorativement à associer l'avant-tournant fictionnel des années quatre-vingt avec la théorisation structuraliste et sémiologique, Barthes inclus, dans le seul but d'annoncer du nouveau, sans vraiment dissocier cette théorisation de la production littéraire concomitante, notamment ricardolienne, mais en récupérant de façon peu rigoureuse les principaux acquis de la réflexion barthésienne en vue d'une approche décomplexée et flexible du fait littéraire contemporain.

6 Denis Hollier revient sans concession sur les enjeux de cette transition. Il se réfère à Logiques de Philippe Sollers, qu'il tient pour bon exemple du devenir de la littérature en ces années « critiques ». Selon lui, la boucle est bouclée :

L'oiseau de Minerve annonçait la clôture : c'est désormais la théorie qui constitue la littérature en ensemble fini et qui, par là même, l'excède. L'exposition de la loi de la série permet d'échapper à la sérialité : au lieu de jouer le jeu, on en expose la règle, la matrice générique, la théorie d'ensemble (Hollier, 1993 : 1021)

Roland Barthes semble y faire allusion dans Roland Barthes par Roland Barthes :

Beaucoup de textes d'avant-garde (encore impubliés) sont incertains : comment les juger, les retenir, comment leur prédire un avenir, immédiat ou lointain ? Plaisentils? Ennuient-ils? Leur qualité évidente est d'ordre intentionnel : ils s'empressent de servir la théorie. Cependant cette qualité est aussi un chantage (un chantage à la théorie) : aimez-moi, gardez-moi, défendez-moi (...) (Barthes, 1975 : 58)

8 Cette période théorisante s'est d'ailleurs elle-même vue mise en fiction par l'une de ses actrices majeures dans Les Samouraï (Kristeva, 1990). Mais, Denis Hollier rappelle que cette génération critique "ne survit plus que sous protectorat universitaire », ou par son expatriation dans les universités américaines, où elle régnera sous le label postmoderne jusqu'à l'épuisement du prestige des sciences humaines françaises, emblématiquement traduit par les déboires, à un moment donné, de la collection «Que sais-je?» (Hollier, 1993 : 1021ss). Désormais, le métalangage appliqué à la description des mécanismes narratifs et aux «notions » utilisées en littérature, fera souvent l'objet de caricatures et de critiques pointant des élucubrations et des exagérations diverses. On constate qu'on est allé trop loin.

On se souvient de certaines critiques adressées jadis par Georges Mounin et Tzvetan Todorov au discours des essais de Roland Barthes, notamment en matière de sémiologie. Elles concernaient un prétendu abus ou des erreurs de transposition des schèmes saussuriens au fait littéraire. Alors, de là à parler d'imposture? Alain Robbe-Grillet rapporte dans Le Miroir qui revient l'obsession éprouvée par Barthes de rester pour la postérité comme un imposteur : 
Roland Barthes (...) semblait, dans la dernière partie de son existence, hanté par l'idée qu'il n'était qu'un imposteur; qu'il avait parlé de tout, de marxisme comme de linguistique, sans avoir rien su vraiment (Robbe-Grillet, 1984 : 63s) coup médiatique et académique avec le fameux Alan Sokal et Jean Bricmont (1997). Raison pour laquelle il devient possible de relire Barthes, surtout en vue d'une approche critique de la littérature contemporaine, à partir de l'intériorisation opérée par la théorisation ; c'est-à-dire en connaissance de cause ou, pour rester barthésien, dans une ouverture sur le « désapprentissage » (cf. Barthes, 1978) serein et synthétique, en somme, décomplexé, et au risque du sens commun. «Paradoxal», pour quelqu'un qui abhorrait la doxa : «(...) contre la Doxa, il faut revendiquer en faveur du sens (...) » (Barthes, $1975: 75$ et 90).

Rappelons, à cet égard, l'essai d'Antoine Compagnon intitulé Le Démon de la Théorie. Littérature et sens commun (1998) dont l'introduction dresse un bilan somme toute motivant, mais problématique pour la recherche littéraire, voire pour la fiction en soi. Dans le «Que reste-t-il de nos amours? » qui ne prélude pas pour autant à un requiem, d'autant moins que «(...) les réponses passent et les questions restent» (Compagnon, 1998 : 15) -, Compagnon avance des constats pertinents pour notre propos, et sur lesquels s'appuie implicitement la théorisation du contemporain: «La théorisation s'est institutionnalisée, elle s'est transformée en méthode» (idem: 11). Et Compagnon de rappeler, non sans humour, qu'« il est impossible aujourd'hui de réussir à un concours sans maitriser les distinguos subtils et le parler de la narratologie ». Voilà donc la théorie « (...) casée, inoffensive, elle attend les étudiants à l'heure dite (...)» (idem : 12). Le statut modeste, presque tâtonnant, de la critique universitaire, revuistique ou journalistique après le tournant opéré par la fiction en langue française ces deux dernières décennies s'est converti en autocritique que les nouvelles revues littéraires reproduisent à la faveur du commentaire sur la fiction. Par exemple, en 1989, Claude Prévost salue le renouveau de la fiction placée "au-delà du soupçon ». Mais on lit déjà en filigrane l'appel à un renouveau de la critique, à laquelle il assigne un rôle discret, "profil bas ", après deux décennies théoriques, polémiques et intolérantes: «la tâche du 'critique' me par[aît] consister à proposer des lectures, au lieu de balancer des verdicts comme un juge d'instance (...)» (Prévost, 1989 : 518). Sans viser qui que ce soit en particulier, les propos de Prévost s'appliquent aussi à un certain penchant apodictique barthésien. Rappelons le démarrage du Degré zéro de l'Écriture : «On sait que la langue est un corps de prescriptions et d'habitudes, communs à tous les écrivains d'une époque » (Barthes, 1953 : 17).

De son côté, Yves Baudelle (1993) passe en revue les mouvances de la critique suscitées par la modernité, et qui seraient contraintes d'infléchir et d'assagir leurs certitudes théoriques. D'abord, la critique marxiste et son approche sociocritique du roman, délaissée depuis le discrédit du marxisme politique. Ensuite, l'école psychanalytique, dont les explorations de l'inconscient demeurent un défi pour l'étude du texte, mais que l'on sent acculée à la modestie conceptuelle une fois rattrapée par le sens commun. Elle continue toutefois d'être opératoire dans l'approche symbolique de certains textes (Durand).

13 La critique thématique aussi, qu'elle renvoie spécifiquement à la phénoménologie de l'imagination appliquée à un thème (Bachelard), ou à une attention portée à la thématique des perceptions sensorielles, représente une part considérable des sujets de thèse. Barthes semble comprendre, mais relativise le plaisir de ce « lire-rêver » : 
Avec Bachelard, c'est toute la poésie (comme simple droit de discontinuer la littérature, le combat) qui passe au crédit du Plaisir. Mais dès lors que l'œuvre est perçue sous les espèces d'une écriture, le plaisir grince, la jouissance pointe et Bachelard s'éloigne (Barthes, 1973 : 61) l'on aurait intériorisés, de la critique formaliste : la sémiologie (Barthes), la narratologie (Greimas, Adam) ; la poétique des textes, laquelle prend la relève, avec Gérard Genette ou Tzvetan Todorov, de l'ancienne rhétorique, et continue de se pencher sur la description théorique et formelle des lois littéraires et des techniques narratives, et suscite nombre de thèses de doctorat, notamment en littérature française contemporaine. recouvrent une nouvelle légitimité à la faveur du retour du monde et du référent dans la fiction narrative, la question, - intériorisée, certes -, des instances narratives demeure intacte dans la perspective critique du texte littéraire, mais son approche se serait assagie, tant il est clair que la théorie n'épuise pas la lecture de l'œuvre. Antoine Compagnon le rappelle en évoquant Barthes, justement : « Il y a une vérité de la théorie, qui la rend séduisante, mais elle n'est pas entièrement théorisable » $(1998: 278)$. résume et intériorise pour tous publics, - universitaire compris -, les grandes lignes de la pensée théorisante de Roland Barthes (Évrard et Tenet, 1994). On ne le ressassera jamais assez, l'écriture advient chez Barthes dans la foulée du signe saussurien : «La langue est donc en deçà de la Littérature. Le style est presque au-delà (...)» (Barthes, 1953 : 9). En effet, le style y assume un caractère vertical et résulte d'un "phénomène d'ordre germinatif » (idem : 20) en conflit avec l'horizontalité de la langue, négative, et « limite initiale du possible». Barthes défrichait ainsi le terrain où l'écriture, loin de tout engagement ou référentialité, se retrouve dans son dénuement formaliste, confrontée au Social et à la Bibliothèque. Elle apparaît, dès lors, en tant que «compromis entre une liberté et un souvenir, elle est cette liberté souvenante (...)» (idem : 28).

Ce discours théorique, - qui semble pour beaucoup d'entre nous acquis, voire intériorisé -, implique la reconnaissance d'un héritage et inscrit Roland Barthes dans la transition chronologique et critique vers le contemporain. Il devient situable, mais aussi transversal. Pascal Ory revient sur ce passage vers le contemporain en évoquant la sortie de scène des grandes figures tutélaires du champ littéraire des années soixante-dix: Sartre, Althusser, Barthes, Lacan, pour en conclure à «(...) la fin d'une certaine conception de la 'modernité' dont on connaît la forme artistique (de l'inspiré à l'avantgarde) (...)» (1983: 244s.). Et Ory de s'interroger sur la nature de ces mutations: décadence ou versant (idem : 245) ? Ceci étant, «figures tutélaires » ne veut pas forcément dire « intellectuels », dont Régis Debray ou encore Michel Winock actent la fin, justement au tournant des années quatre-vingt (Debray, 2000 ; Winock, 1997).

Pascal Ory (1983) rappelle l'implication revuistique autour de Tel Quel, Change, Le Chemin, Digraphe, Txt ; souvent secouée par la contestation et la dissidence, alimentée par les travaux de Jacques Derrida, Roland Barthes, Jean Ricardou ou Julia Kristeva, et qui poursuivait un projet totalisant ( $\mathrm{Tel}$ Quel convoque, d'ailleurs, en sous-titre plusieurs discours sociaux), mais aux conséquences extrémistes sur la littérature.

Dans son second tome d'histoire culturelle contemporaine, Pascal Ory (1989) reflète la perplexité face aux caractéristiques novatrices de l'écriture française après la Textualité, c'est-à-dire la « nouvelle fable » (idem : 222). Selon lui, et en clin d'œil à Barthes, l'écriture 
française renouerait avec le "plaisir du texte». Ory va même plus loin: «l'époque retrouvait sans honte le Plaisir du texte " (ibid.). Elle voit aussi l'évolution de Roland Barthes, passant aux aveux, renouant avec la pluralité des lectures, regardant d'un bon œil un retour en force des matériaux autobiographiques. Ory ne manque pas non plus de souligner le tour pris par la critique et l'histoire littéraires après 1980 (c'est-à-dire après le décès de Barthes), avec notamment le succès de l'analyse génétique et la réhabilitation du biographisme.

Or, la mitoyenneté de l'écriture se joue précisément dans l'entre-deux du champ littéraire, tel que Roland Barthes, - encore lui -, l'avait défini dans sa tentative de dégagement historique, autour de Flaubert surtout, d'une autonomie de l'écrivain vis-àvis du corps social ; qui est avant tout « autonomie de l'art» (Bourdieu, 1992), et dont la modernité est foncièrement tributaire.

21 À partir de la fin des années quatre-vingt, - comme l'a bien remarqué Fieke Schoots (1997), notamment pour les éditions de Minuit -, la critique décèle l'émergence d'un nouveau champ littéraire après la Textualité ; d'où le profond « désarroi » éprouvé par bien des éditoriaux de l'époque. En effet, la critique a le sentiment de se trouver sur un «terrain vague» (idem: 9) ou, selon l'expression de Jean-Pierre Salgas, « une zone frontière où l'œuvre manifeste sa solidarité ou ses défiances à l'égard de la bibliothèque et de l'Histoire » (1997: 10), c'est-à-dire à une intersection.

Salgas semble avoir saisi la portée opératoire de la notion bourdieusienne du champ sur le paysage littéraire actuel : «mon arrière-pensée va plutôt, outre le premier Barthes, au Pierre Bourdieu des Règles de l'art (...), à une réflexion en terme de 'champ littéraire' insécablement esthétique et institutionnel» (idem: 43). La perte d'une certaine autonomie du champ littéraire contemporain serait à mettre sur le compte de l'apesanteur d'un champ désormais sans bords ni centre.

En effet, trois notions balisent, selon lui, ce nouveau territoire d'écriture. Respectivement, la définition qu'en donne le Roland Barthes du Degré zéro de l'écriture induisant l'approche intransitive du texte, le paratexte collectif selon Gérard Genette, et l'intertextualité d'après Julia Kristeva. Habituée à l'opacité affichée de l'écriture, la critique s'ouvre soudain aux indices d'une recherche en "dépli», synthétique et citationnelle, mais qui ne peut faire l'économie des acquis précédents ou qui s'y heurte forcément.

Parmi ces critiques, citons Jean-Jacques Amette, lequel n'hésite pas à nommer cette génération de " postmoderne ». En font partie des nouveaux néo-romanciers minuitards tels que Jean-Philippe Toussaint, Jean Echenoz, Bertrand Visage ou encore Patrick Deville (1989) dont l'écriture suggère la synthèse, l'intégration, voire le recyclage de la tradition et de la modernité :

(...) l'écriture blanche durassienne n'est jamais loin; ni Lacan, ni les linguistes associés, ni les cours de facs structuralistes. Mais ces écrivains ont transformé cette culture universitaire grâce à un humour adolescent, une impertinence intellectuelle que n'avaient ni les Roland Barthes ni les Robbe-Grillet (ibid.)

Mais le renvoi le plus remarqué à Barthes dans la théorisation du renouveau romanesque concerne son intrusion implicite dans la conception critique de l'autofiction; ce genre romanesque hybride associant la référence biographique à l'affichage assumé de la fiction, notamment dans le péritexte et le paratexte. Roland Barthes avait déjà fait allusion dans Le Plaisir du texte au « fictif de l'identité » (1973 : 98), avant de faire paraître en 1975 Roland Barthes par Roland Barthes, texte à l'écriture subtile, et dont le péritexte 
annonce la démarche subversive, parce qu'" autobiographique ", alors même que les recherches sur le genre autobiographique progressent et gagnent en systématisation, ou en dérogation (Lejeune, 1975). Rappelons les mots de Barthes: "Tout ceci doit être considéré comme dit par un personnage de roman» (1975: page de garde). Propos étonnants si l'on considère sa théorisation textuelle précédente.

Le passage le plus retentissant à l'autobiographie sera opéré par l'un des représentants les plus prestigieux du Nouveau Roman lorsque, en 1984, Alain Robbe-Grillet surprend avec la publication d'un premier tome d'une trilogie autofictionnelle, Le Miroir qui revient dont l'incipit prend des allures de provocation ou de rétractation: «Je n'ai jamais parlé d'autre chose que de moi. Comme c'était de l'intérieur, on ne s'en est guère aperçu " (1984: 9). Comme l'a bien souligné Aron Kibédi Varga, cette volte-face prônait une relecture autoréférentielle d'autres textes issus du Nouveau Roman, voire de la Textualtité (1986 : 11).

L'entreprise autobiographique de Robbe-Grillet nargue les définitions de plus en plus canoniques dégagées par les études en autobiographie. En effet, le roman expressément « autobiographique » de Robbe-Grillet affiche ouvertement la subversion et l'ambiguïté en indiquant "romanesques" dans le péritexte. La fausse piste ou les faux indices péritextuels deviennent une solution de plus en plus prônée, - dira Umberto Eco pour annoncer une autobiographie en mal de référentialité. Désormais, pour reprendre Eco, « un titre doit embrouiller les idées, non les embrigader » (Есо, $1985: 11)$.

Il est évident que la démarche de Robbe-Grillet répond quelque part au Roland Barthes par Roland Barthes, dont elle partage aussi la stratégie. Le Miroir qui revient ne correspond-t-il pas à un projet longuement mûri depuis 1976 d'un " Alain Robbe-Grillet par lui-même »? Roland Barthes par Roland Barthes, paru dans la collection de Denis Roche «Écrivains de toujours " au Seuil, s'avère une autobiographie fragmentaire ; un Roland Barthes sur luimême, mais par écriture interposée. À sa parution, ce texte est reçu comme un exercice ludique, une sorte de gag, notamment par son aspect de pastiche. Il calque le format de la collection, surtout par la place accordée à la photo, par exemple l'album commenté ( $c f$. Viart et Vercier, $2005: 58,277)$. On pense à l'autobiographie, mais bien vite, on s'aperçoit qu'il s'agit d'un tout autre projet. Barthes s'y désigne par «il», "vous», ou par ses initiales R. B. dans un monologue fragmenté où se déclinent les soucis théoriques de toujours : le langage, l'écriture, la politique, l'inconscient, mais aussi le corps et le désir; le tout établi en entrées d'un dictionnaire, ou glossaire narratif qui va de "Actif/ réactif " à «Le monstre de la totalité ». On se perd dans les pronoms, mais finit par se retrouver dans le projet global.

Ce qui est d'ores et déjà frappant dans cet engouement pour l'écriture de soi, et dans cet éclairage plus ou moins autobiographique porté rétrospectivement par les nouveaux romanciers ou écrivains telquelliens sur leur écriture précédente, c'est le fait que le retour du sujet narratif ne se produit pas sur le mode strictement référentiel, mais plutôt par le truchement d'une élaboration fictionnelle, par le « fictif » identitaire barthésien. Le soupçon des décennies antérieures aura donc décisivement affecté les stratégies de la représentation du moi.

30 Dans l'entrée "Moi, je », de Roland Barthes par Roland Barthes, la question est explicitée : "Cependant, aujourd'hui, le sujet se prend ailleurs, et la 'subjectivité' peut revenir à une autre place de la spirale: déconstruite, désunie, déportée, sans ancrage: pourquoi ne parlerais-je pas de 'moi', puisque 'moi' n'est plus 'soi'?» (Barthes, 1975: 171) Cette 
nouvelle tendance, remarquée chez Roland Barthes, ne manque pas d'être glosée par les principaux théoriciens et anthologistes de la littérature française contemporaine. Aussi, Bruno Blanckeman introduit-il sa "Mise en perspective historique» de la fiction contemporaine par une citation solennelle de Barthes. Il s'agit de la définition que l'auteur de Leçon donne de la littérature au Collège de France en 1977, et qu'il applique, en l'actualisant, à la littérature contemporaine :

Il [Barthes] la [la littérature] saisit à la lettre, dans la détermination polymorphe de sa matière, la langue, et de la délimitation labile de ses manières, des expériences d'écriture. Ce graphe, dans lequel Barthes reconnaît la silhouette de la littérature, est éminemment mobile : tracé par le temps, il se redessine périodiquement (...) (Blanckeman, $2000: 9$ )

Blanckeman invoquera également Barthes pour baliser un de ses propos majeurs: l'illustration des mécanismes narratifs et des enjeux identitaires de l'autofiction comme genre émergent, notamment chez Hervé Guibert (idem : 23).

Le Viart et Vercier parle d'un «souci de soi » $(2005: 26)$ qui aurait affecté Barthes dans l'écriture de son autobiographie par ordre alphabétique. Le chapitre intitulé «Variations autobiographiques ", qui caractérise les modalités du soi-disant retour du sujet dans le panorama fictionnel français, évoque Barthes pour souligner que « Au cours de la période contemporaine l'interrogation s'est déplacée : elle ne porte plus sur le sujet dont on fait le récit mais sur celui qui entreprend ce récit » (idem : 46), mais pour l'approche proprement critique des nouveaux territoires d'écriture, la théorisation barthésienne se voit plutôt évitée ou mise au défi, notamment au profite du fait divers et du refus de la clôture : «Ces textes échappent (...) à la réception 'structurale' autrefois théorisée par Roland Barthes dans les Essais critiques (...) » (idem : 241). Plus loin, Barthes est remémoré pour son éloge du haïku dont on retrouve la technique ou la propension chez certains romanciers contemporains (idem : 272).

De son côté, Jean Bessière, dans Le Roman contemporain ou la problématicité du monde (2010) place le roman actuel "face à la tradition", et invoque Roland Barthes pour rendre compte de l'interaction théorie et écriture romanesque dont la fiction serait quelque part tributaire :

La contrepartie de ces types de théories ou de thèse se lit dans le discours sur la fin du roman. Le roman est ultimement assimilé à l'écriture : il est sans trait distinctif. Par un jeu réciproque inévitable, l'écriture ne contredit pas le roman : elle peut conduire à l'entreprise romanesque. L'obsession qu'eut Roland Barthes d'écrire un roman traduit cela même (idem : 41)

Bessière met un bémol assagi aux thèses structuralistes qui postulaient la méfiance face au réalisme :

Cela fait comprendre pourquoi le roman réaliste ne peut être tenu pour, quoi qu'en ait pensé Roland Barthes, pour une illusion : il ne peut l'être parce qu'il participe de l'anthropoiesis de l'individualité et qu'il illustre la dualité du singulier et du paradigmatique. $\mathrm{Ou}$, en d'autres termes, que le roman réaliste n'entend pas se donner comme le jumeau de la réalité (idem : 281)

Pour Jacques Lecarme, l'autofiction peut être vue dans deux sens distincts. Au sens strict, elle réfère au récit de faits réels. La fiction porte alors, non sur le contenu évoqué, mais sur le processus d'énonciation, sur sa mise en récit. Le Roland Barthes par Roland Barthes s'inscrit, selon lui, dans cette première catégorie; tandis que, au sens large, l'autofiction associe le vécu à l'imaginaire. Dans ce cas, la fiction affecte substantiellement le contenu narré. Ce serait le cas de la trilogie « romanesque » d'Alain Robbe-Grillet. 

s'octroie le plaisir du texte dans le jeu même de l'écriture dont il n'a jamais cessé de caractériser le tissu. L'heure est aux affirmations hypothétiques, aux tâtonnements théoriques comme en témoigne cette notion tardive du texte recevable, " texte ardent ", que Roland Barthes place à côté du lisible et du scriptible dans Roland Barthes par Roland Barthes quand «il» se réfère aux «amis»: «La question m'est posée par le développement de mille péripéties d'amitié. Ainsi s'écrit au jour le jour un texte ardent, un texte magique, qui ne finira jamais, image brillante du Livre libéré » (Barthes, 1975 : $68 s)$.

Franck Évrard et Éric Tenet disent de ce sujet mis en (auto)fiction qu'il :

(...) est donc 'effet de langage'. Ce moi qui n'est pas moi, disséminé dans le texte est un sujet imaginaire, 'un être de papier'. Ce sujet écrit n'existe pas hors de l'espace d'un texte dont l'auteur n'est finalement qu'un personnage parmi d'autres (1994: 105) ;

ce qui fait de Roland Barthes par Roland Barthes :

une autobiographie au neutre où l'auteur écrit son existence en creusant la distance entre lui et lui-même, décollant l'imaginaire de son support, donnant des traits de langage et non un portrait intime, parlant de lui 'comme d'un peu mort' (idem : 108)

Roland Barthes se trouve donc intrinsèquement convoqué dans la conceptualisation théorique de la transition vers la fiction contemporaine. À cet égard, Sémir Badir décrit, après Roland Barthes, l'émergence d'un «troisième degré " pour l'écriture contemporaine, qui ne serait plus forcément dialectique, mais fondé sur «une incertitude, (...) une inconnue " et voué à parcourir "le chemin du milieu», et à embrasser l'éthique de la relation (Badir, 1993 : 18s). Reprenant en cela le rôle de la culture à l'ère postmoderne chez Jean-François Lyotard, Badir assigne à la postmodernité littéraire une fonction lucide de résistance ou, comme chez Roland Barthes, une vocation $\mathrm{au}$ « désapprentissage » totalement lucide ; une lucide (non-) ignorance du passé.

En outre, la théorisation du renouveau romanesque au point de bascule des années quatre-vingt met en exergue la place de la réception du texte et le statut du lecteur dans un contexte, lui aussi, nouveau. Là encore, les acquis de la théorie structuraliste et de la sémiologie bathésienne s'avèrent marquants, d'autant plus que le liseur est mis à l'honneur dans la théorisation de Barthes par la nuance entre texte lisible (lu, mais nonsusceptible de réécriture) et le texte scriptible ; celui qui, au contraire, « (...) fait sortir le lecteur du rôle assigné par l'idéologie [et qui] se dérobe au résumé tant il apparait comme une exception, une nouveauté » (Évrard et Tenet, 1994 : 76).

40 Comme l'a relevé Yves Baudelle, la nouvelle critique se fonde avant tout sur le refus de "la primauté de la conscience créatrice », en faveur d'une « lecture polysémique », de la "prolifération infinie des significations» soutenue par Barthes dans le concept de « lecteur pluriel », lui-même quelque part « producteur de texte » (cf. idem : 78).

Or, dans Le Démon de la théorie, Antoine Compagnon répond implicitement à deux interrogations : rétablir un rôle équilibré du lecteur, dont on aperçoit la résistance malgré la théorie, et définir la nouvelle posture de ce lecteur face aux caractéristiques scripturales du roman contemporain. Compagnon passe en revue les aléas du lecteur pris au piège de la théorie littéraire structuraliste, hantée par la description d'un texte marqué par la neutralité et l'autonomie. L'auteur de La Littérature, pour quoi faire ? regrette que le lecteur y soit réduit à la condition d'« intrus », étranger au fonctionnement interne du texte. 
D'où les apories d'une telle approche du lecteur et que Compagnon souligne :

Mais que dire du lecteur qui n'a pas reçu cette initiation traditionnelle au roman? Ou encore le roman contemporain, parfois qualifié de postmoderne, fragmentaire et déstructuré ? Sa conduite est-elle encore réglée par une quête de cohérence sur le modèle du roman réaliste? (Compagnon, $1998: 164 \mathrm{~s}$ )

Qui plus est, le caractère ludique, ironique, citationnel et surcodé du roman contemporain, du fait de son schéma narratif fragmentaire, juxtaposé, et en clin d'œil visà-vis de l'héritage littéraire classique et moderne, met en jeu « (...) un extérieur qui se veut littéraire, il ne s'adresse qu'à des déjà lecteurs »(Demoulin, $1997: 11$ ).

À ce titre, Nicolas Ancion dans Écritures réapprécie le rôle du lecteur en convoquant librement Roland Barthes et sa vision du lecteur dans $S / Z$ ou Le Plaisir du texte, et sur les nuances notionnelles de "texte-lecture de plaisir», "texte-lecture de jouissance». Ancion dégage la nature changeante de la lecture dans son contexte postmoderne. Pour ce faire, il rappelle que la lecture littéraire se joue entre le texte et le lecteur, et implique un "protocole de lecture", par lequel le lecteur assigne une fonction au texte étant donné « le but que le lecteur confère à sa lecture, et qui ressortit aussi bien à la nature du texte qu'à la compétence lectrice » (Ancion, 1993 : 30s).

L'infinitude de protocoles de lecture suggère leur regroupement en «modalités protocolaires » (idem : 32), un facteur socio-historique renvoyant à la consolidation des systèmes scolaires obligatoires et à l'émergence d'un champ littéraire autonome selon la conception bourdieusienne. Et Nicolas Ancion d'énumérer les trois modalités protocolaires ayant cours durant la modernité littéraire.

D'abord, la lecture en tant que plaisir, c'est-à-dire, dit Ancion, la reconnaissance et recréation du sens préexistant et indéfini du texte. La critique textuelle et les revues littéraires la pratiqueraient à l'envi. Ensuite, la lecture en tant que devoir, issue de l'institution de la scolarisation obligatoire. Enfin, la lecture en tant que «jouissance », à savoir celle qui attribue davantage d'importance à l'acte de lecture en soi qu'au texte lu. Cette modalité de lecture cherche dans un texte un discours neuf, non encore exprimé.

À ce propos, Roland Barthes avait signalé ces nuances théoriques applicables au champ littéraire actuel, et dont la théorisation du renouveau contemporain se saisit de façon opportuniste. L'écrivain de plaisir et son lecteur relèvent d'un «parler sur » (1973: 37s), où le langage se superpose à la parole, puisque «ce plaisir peut être dit : de là vient la critique » (ibid.). En revanche, l'écrivain de jouissance, et son lecteur, sont aux prises avec un "texte intenable», "texte impossible», renvoyant à un "parler en» fonctionnant " hors de toute finalité imaginable » (ibid.).

Entre plaisir et jouissance, il est une "marge d'indécision" où s'ouvre une zone d'indétermination, entre la prévisibilité du texte et le travail de lecture : «ce lecteur, il faut que je le cherche, [que je le 'drague'], sans savoir où il est » (idem: 11). Un espace de jouissance est alors créé : «Ce n'est pas la 'personne' de l'autre qui m'est nécessaire, c'est l'espace (...)» (ibid.). La jouissance réfère à cette atopie du texte et de sa lecture, en situation « hors-critique » et « hors-plaisir » (idem : 37 ).

Ancion en conclut que la lecture comme plaisir, le «parler sur » barthésien a largement dominé le discours littéraire moderne. Toutefois, il accuse de plus en plus les symptômes d'une crise dont on devine déjà quelques aspects : le franchissement d'un seuil critique avec le Nouveau Roman et le théoricisme pour ce qui est du «plaisir » escompté à la lecture d'un texte; la déhiérarchisation des genres littéraires et la relativisation de la 
légitimité littéraire des textes (leur rapport à la culture lettrée), ainsi que la réhabilitation intégrale du rôle créatif du lecteur.

Dès lors, la mutation détectée à cette période revendique une liberté esthétique ainsi que l'affirmation de droits du lectorat. Il suffit de considérer l'impact de l'« effet Pivot » et la médiatisation de la littérature dans "Apostrophes ", lesquels dissolvent les questions: «Qu'est-ce que la littérature? ? et « Que peut la littérature?».

51 Tout aussi révélateur de cette tendance, signalons le succès éditorial international, dès sa parution en 1992, de Comme un roman de Daniel Pennac que deux idées fortes balisent. D'une part, la lecture devient de plus en plus incompatible avec le devoir et le dogme : « le verbe lire ne supporte pas l'impératif » (Pennac, 1992: 13). D'autre part, le lecteur y met forcément du sien : « la lecture est un acte de création permanente » (idem : 27).

Ainsi, plus qu'hésiter entre lecture de plaisir et lecture de jouissance, la théorisation contemporaine nivèle le prestige des modalités de lectures héritées de la modernité; et d'autre part, tout en invoquant Roland Barthes, souligne un éventail de pratiques de lecture axées sur la "jouissance ", fût-ce au risque de se taire: "Grande jouissance de lecteur, ce silence d'après la lecture » (idem : 20).

C'est dire combien Barthes s'impose toujours sur le discours critique, combien il s'avère incontournable dans la lecture de la fiction contemporaine et combien on s'en approprie à tort ou à raison. On distingue toujours les écrivains des écrivants, le passé simple, dont Barthes se méfiait (1953: 46-49), résiste, et parfois s'affiche, le subjonctif, parfois imparfait, est remarqué dans la fiction contemporaine (idem : 108s). Alors?

Barthes se méfiait, certes, de certaines modalités du nouveau : «(...) neuf fois sur dix, le nouveau n'est que le stéréotype de la nouveauté » (Barthes, 1973 : 65), mais la question de l'écriture en littérature contemporaine demeure : «(...) le style est le commencement de l'écriture : même timidement, en s'offrant à de grands risques de récupération, il amorce le règne du signifiant » $(1975: 80)$.

\section{BIBLIOGRAPHIE}

AMETTE, Jacques Pierre (1989). « Le nouveau 'nouveau roman », in Le Point, 16 janvier.

A.A.V.V. (1992). Autofictions \& $C^{i e}$. Colloque de Nanterre, sous la direction de Serge Doubrovsky, Jacques Lecarme et Philippe Lejeune, RITH, $n^{\circ} 6$.

ANCION, Nicolas (1993). « La lecture littéraire, entre post- et modernité », in Écritures, $n^{\circ} 5$.

BADIR, Sémir (1993). « Vers la postmodernité. Retour à Baudelaire », in Écritures, n 5.

BAUDELlE, Yves (1993). «Les grandes lignes de la littérature française depuis 1945 », in Fr. Baert et D. Viart (dir.). La Littérature française contemporaine. Questions et perspectives. Louvain : Presses Universitaires de Louvain.

BARTHES, Roland (1953). Le Degré zéro de l'écriture. Paris : Le Seuil.

BARTHES, Roland (1973). Le Plaisir du texte. Paris : Le Seuil 
BARTHES, Roland (1970). L'Empire des signes. Paris : Skira.

BARTHES, Roland (1978). Leçon. Paris : Le Seuil.

BESSIERE, Jean (2010). Le Roman contemporain ou la problématicité du monde. Paris : PUF.

BLANCKMAN, Bruno (2000). Les Récits indécidables : Jean Echenoz, Hervé Guibert, Pascal Quignard. Paris : Presses Universitaires du Septentrion.

BOURDIEU, Pierre (1992). Les Règles de l'art. Genèse et structure du champ littéraire. Paris : Le Seuil. COMPAGNON, Antoine (1998). Le Démon de la théorie. Littérature et sens commun. Paris : Le Seuil. DEBRAY, Régis (2000). I.F. suite et fin. Paris : Gallimard.

DEMOULIN, Laurent (1997). « Génération innommable », in Textyles, $\mathrm{n}^{\circ} 14$.

ECO, Umberto (1985). Apostille au Nom de la rose. Paris : Grasset.

ÉVRARD, Franck et Tenet Éric (1994). Roland Barthes. Paris: Bertrand-Lacoste.

HOLLIER, Denis (1993). « Comment peut-on être français?», in Denis Hollier (dir.). De la littérature française. Paris : Bordas.

KRISTEVA, Julia (1990). Les Samouraïs. Paris : Gallimard.

LEJEUnE, Philippe (1975). Le Pacte autobiographique. Paris : Le Seuil.

ORY, Pascal (1983). L'entre-deux-Mai. Histoire culturelle de la France. Mai 1968-Mai 1981. Paris : Le Seuil. ORY, Pascal (1989). L'Aventure culturelle française. Paris : Flammarion.

PREVOST, Claude (1989). « Au-delà du soupçon », in La Quinzaine Littéraire, n 532, 16-31 mai.

ROBBE-GRILLET, Alain (1984). Le Miroir qui revient. Paris : Minuit.

SALGAS, Jean-Pierre (1997). « 1960-1990 : romans mode d'emploi, suivi de Post-scriptum 1997 », in Roman français contemporain. Paris : Ministère des Affaires Etrangères, Sous-Direction du Livre et de l'Ecrit.

SHоOTS, Fieke (1997). « Passer en douce à la douane ». L'écriture minimaliste de Minuit. Amsterdam/ Atlanta : Rodopi.

SOKAL, Alan et Bricmont, Jean (1997). Impostures intellectuelles. Paris : Odile Jacob. Varga, Aron Kibédi (1986). « Récit et postmodernité », in Littérature et Postmodernité. Amsterdam / Adanta : Rodopi.

VIART, Dominique et Vercier, Bruno (2005). La Littérature française au présent. Héritage, modernité, mutations. Paris : Bordas.

WINOCK, Michel (1997). Le Siècle des Intellectuels. Paris : Le Seuil.

\section{RÉSUMÉS}

Le renouveau romanesque français dans le tournant des années 1980, et sa théorisation, convoquent Roland Barthes en tant que producteur d'un métalangage narratologique intériorisé aussi bien par les écrivains dans leur pratique scripturale que par les critiques littéraires, lequel a ainsi subi une appropriation et une assimilation, d'autant plus que son discours et ses concepts métaphoriques s'y prêtent. Cet article passe en revue l'impact théorique de Barthes sur les discours critiques du renouveau romanesque en langue française. 
The French fictional renewal in the early 1980s and its theorisation, summons Roland Barthes as a producer of a narratological internalized metalanguage both by the writers and the book reviewers, that has gone through an appropriation and assimilation, especially as his discourse and his metaphorical concepts are conducive to it. This paper reviews the theoretical Barthes impact on the critical discourse of French fictional renewal.

INDEX

Keywords: Barthes (Roland), theorisation, French literature, renewal, novel

Mots-clés : Barthes (Roland), théorisation, littérature française, renouveau, roman

\section{AUTEUR}

\section{JOSÉ DOMINGUES DE ALMEIDA}

ILC Margarida Losa

Universidade do Porto

jalmeida@letras.up.pt 